\title{
Placenta-derived extracellular vesicles induce preeclampsia in mouse models
}

Haematologica 2020

Volume 105(6):1686-1694

\author{
Cha Han, ${ }^{1,2^{*}}$ Chenyu Wang, ${ }^{3 *}$ Yuanyuan Chen, ${ }^{1}$ Jiwei Wang, ${ }^{4} \mathrm{Xin} \mathrm{Xu},{ }^{2}$ Tristan \\ Hilton, ${ }^{2}$ Wei Cai, ${ }^{3}$ Zilong Zhao, ${ }^{5}$ Yingang Wu, ${ }^{6} \mathrm{Ke} \mathrm{Li},{ }^{1}$ Katie Houck, ${ }^{2}$ Li Liu, ${ }^{5}$ Anil \\ K. Sood, ${ }^{7}$ Xiaoping Wu, ${ }^{2}$ Fengxia Xue, ${ }^{1}$ Min Li, ${ }^{3}$ Jing-fei Dong ${ }^{2,8}$ and Jianning \\ Zhang ${ }^{5}$
}

${ }^{1}$ Department of Obstetrics and Gynecology, Tianjin Medical University General Hospital, Tianjin, China; ${ }^{2}$ Bloodworks Research Institute, Seattle, WA, USA; ${ }^{3}$ Institute of Pathology, School of Medical Sciences and Gansu Provincial Key Laboratory of Preclinical Study for New Drug Development, Lanzhou University, Lanzhou, China; ${ }^{4}$ Department of Neurosurgery, Tianjin Key Laboratory of Cerebral Vascular and Neurodegenerative Diseases, Tianjin Huanhu Hospital, Tianjin, China; ${ }^{5}$ Department of Neurosurgery, Tianjin Medical University General Hospital and Tianjin Neurological Institute, Tianjin, China; ${ }^{6}$ Department of Neurosurgery, the First Affiliated Hospital, Division of Life Sciences and Medicine, University of Science and Technology of China, Hefei, China; ${ }^{7}$ Department of Gynecologic Oncology and Reproductive Medicine, Division of Surgery, MD Anderson Cancer Center, University of Texas, Houston, TX, USA and ${ }^{8}$ Division of Hematology, Department of Medicine, University of Washington, School of Medicine, Seattle, WA, USA.

${ }^{\star} \mathrm{CH}$ and $\mathrm{CW}$ contributed equally as co-first author.

\section{Correspondence: \\ MIN LI \\ P}

limin@|zu.edu.cn

JIANNING ZHANG

jianningzhang@hotmail.com

JING-FEI DONG

jfdong@bloodworksnw.org

Received: May 6, 2019

Accepted: August 22, 2019.

Pre-published: August 22, 2019.

doi:10.3324/haematol.2019.226209

Check the online version for the most updated information on this article, online supplements, and information on authorship \& disclosures: www.haematologica.org/content/105/6/1686

(C)2020 Ferrata Storti Foundation

Material published in Haematologica is covered by copyright. All rights are reserved to the Ferrata Storti Foundation. Use of published material is allowed under the following terms and conditions:

https://creativecommons.org/licenses/by-nc/4.0/legalcode. Copies of published material are allowed for personal or internal use. Sharing published material for non-commercial purposes is subject to the following conditions:

https://creativecommons.org/licenses/by-nc/4.0/leǵalcode, sect. 3. Reproducing and sharing published material for commercial purposes is not allowed without permission in writing from the publisher.

\section{ABSTRACT} reeclampsia is a pregnancy-induced condition that impairs the mother's health and results in pregnancy termination or premature delivery. Elevated levels of placenta-derived extracellular vesicles $(\mathrm{pcEV})$ in the circulation have been consistently associated with preeclampsia, but whether these vesicles induce preeclampsia or are the product of preeclampsia is not known. Guided by a small cohort study of preeclamptic patients, we examined the impact of pcEV on the pathogenesis of preeclampsia in mouse models. We detected pcEV in pregnant C56BL/6J mice with a peak level of $3.8 \pm 0.9 \times 10^{7} / \mathrm{mL}$ at $17-18$ days postcoitum. However, these pregnant mice developed hypertension and proteinuria only after being infused with vesicles purified from injured placenta. These extracellular vesicles released from injured placenta disrupted endothelial integrity and induced vasoconstriction. Enhancing the clearance of extracellular vesicles prevented the development of the extracellular vesicle-induced preeclampsia in mice. Our results demonstrate a causal role of pcEV in preeclampsia and identify microvesicle clearance as a new therapeutic strategy for the treatment of this pregnancy-associated complication.

\section{Introduction}

Preeclampsia is a pregnancy-induced pathology characterized by poor placentation and endothelial dysfunction. Its primary clinical presentations include newonset hypertension and proteinuria that resolve or are significantly improved after delivery or pregnancy termination. ${ }^{1,2}$ Preeclampsia can progress into eclampsia, potentially resulting in maternal and fetal death. Extensive clinical and laboratory studies have demonstrated that preeclampsia is triggered by placenta-derived mediators $^{3}$ produced after placental ischemia and reperfusion injury, which could result from placental spiral artery remodeling disorders. ${ }^{4,5}$ One of these mediators is placenta-derived extracellular vesicles (pcEV), which are released into the maternal circulation, typically reaching a peak level in late pregnancy. ${ }^{6-8}$ These pcEV are constitutively released during normal pregnancy and are necessary for inducing maternal adaptive changes such as tolerance. ${ }^{9}$ However, excessive shedding of pcEV often indicates placental pathologies that contribute to the pathogenesis of preeclampsia. 
Significantly elevated plasma levels of pcEV have been consistently associated with the development and severity of preeclampsia. ${ }^{10,11}$

The pcEV found in patients with preeclampsia are heterogeneous in their cells of origin, size, and cargo contents, and thus possess diverse activities, some of which may not be apparent or detectable in their parental cells. Despite this heterogeneity, extracellular vesicles (EV) from syncytiotrophoblasts are widely used as the surrogate marker for detecting pcEV in the maternal circulation, ${ }^{12,13}$ even though there is no evidence in the literature that these pcEV directly cause preeclampsia. Placental cells vesiculate when the placenta is subjected to ischemic and hypoxic injuries that result in cell apoptosis and tissue necrosis. ${ }^{14-17}$ These injuries can also occur when trophoblasts are unable to infiltrate the wall of the placenta's spiral artery to gradually replace the endothelium in a process called "blood vessel recasting". ${ }^{14-17}$

Once released into the circulation, pcEV can cause endothelial injury, ${ }^{18-20}$ systemic inflammation, ${ }^{21,22}$ and coagulation dysfunction, ${ }^{23}$ all of which are hallmark events of preeclampsia. Despite extensive studies on the causes and mechanisms of placental injuries, key questions remain as to whether pcEV released from injured placenta directly induce the hypertension and proteinuria that define preeclampsia or are merely the products of preeclampsia. If the former holds true, could accelerating or enhancing the clearance of pcEV prevent preeclampsia or reduce its severity? Here we report the results of a study designed to answer these questions by analyzing blood samples from preeclamptic patients, studying new mouse models of preeclampsia, and conducting in vitro experiments.

\section{Methods}

\section{Study of patients}

Pregnant women were recruited from Tianjin Medical University General Hospital after they had given informed consent to participate in the study. Blood samples were collected from patients with preeclampsia at the diagnosis of their condition, before the administration of magnesium sulfate or anti-hypertensive medications, and analyzed for plasma levels of pcEV (Online Supplementary Methods). This study was approved by the Ethics Committee of Tianjin Medical University General Hospital.

\section{Mouse models}

We used three complementary models to study the role of pcEV in the development of preeclampsia (Online Supplementary Methods). The first model was designed to study whether increasing circulating pcEV in pregnant mice induced preeclampsia. For this study, we defined a mouse preeclampsia phenotype by hypertension, proteinuria, and kidney injury. ${ }^{1}$ Blood pressure (BP) was measured at baseline, 17-18 days post-coitum (dpc), and 7-10 days postpartum using a noninvasive mouse tail-cuff BP analyzer (CODA; Kent Scientific Co., Torrington, CT, USA). ${ }^{24}$ Urinary albumin and creatinine concentrations in a pooled urine sample collected over a 24 -h period were measured and their ratio was calculated to define proteinuria (Online Supplementary Methods). The second model was used to study whether pcEV induced hypertension and proteinuria in non-pregnant C57BL/6 female mice. BP was measured $30 \mathrm{~min}$ after the pcEV infusion and $24 \mathrm{~h}$ urine samples were analyzed for proteinuria. The third model was used to specifically investigate the role of EV clearance.

\section{Flow cytometry}

Levels of pcEV in plasma samples from women with a normal pregnancy or preeclampsia were measured using a fluorescein isothiocyanate-conjugated antibody against placental alkaline phosphatase (PLAP; LifeSpan Biosciences, Inc., Seattle, WA, USA). ${ }^{11,14,20,25}$ For the mouse study, we used syncytin as the marker for pcEV because PLAP is not expressed in mouse placenta (Online Supplementary Methods). ${ }^{26}$

\section{Hematologic measurements}

We used three tests to measure the procoagulant activity of pcEV: (i) a phosphatidylserine (PS)-dependent plasma-clotting assay that specifically measured microvesicle-mediated coagulation (Online Supplementary Methods); ${ }^{27}$ (ii) platelet activation; and (iii) detection of plasma levels of the anionic phospholipid PSexpressing EV. Platelet activation and $\mathrm{PS}^{+} \mathrm{EV}$ were detected by, respectively, a phycoerythrin-CD62p antibody (eBiosciences) and allophycocyanin-annexin V (eBiosciences) using flow cytometry. 27,28

\section{Vascular leakage}

We used an Evans blue extravasation test to measure pcEVinduced vascular leakage in vivo (Online Supplementary Methods). ${ }^{28}$ We also measured the ability of pcEV to disrupt the integrity of cultured cells from the mouse endothelial line bEnd.3 (ATCC, Manassas, VA, USA) (Online Supplementary Methods). ${ }^{27,29}$

\section{Microvesicle-induced vasoconstriction and changes in cerebral blood flow}

We measured vascular wall tension using a modified protocol (Online Supplementary Methods). ${ }^{30} \mathrm{We}$ also used non-invasive laser speckle contrast analysis (LASCA) technology to measure the impact of pcEV on cerebral blood flow. Cerebral blood flow was chosen because LASCA cannot accurately measure the blood flow of internal organs such as the kidney without surgery, which would have been a confounding injury that would have been difficult to stratify.

\section{Statistical analysis}

Quantitative data are expressed as percentages for categorical (frequency) variables or as the mean \pm standard error of the mean for continuous variables. For parametric data, a Shapiro-Wilk test was performed to test the distribution of the data. Data were analyzed using a paired $t$-test or one-way or repeated-measures analysis of variance, as specified for each dataset. A $P$ value of less than 0.05 was considered to be statistically significant. For multiple comparisons, the Holm-Sidak method was used to control for family-wise error rate (Sigma plot V. 11.2).

\section{Results}

\section{Placenta-derived extracellular vesicles were detected in preeclamptic women and pregnant mice}

Among the 17 pregnant women (all first pregnancies) recruited into the study, ten were diagnosed as having preeclampsia between 28 and 38 weeks of gestation. Their information is listed in Table 1. Patients were excluded from the study if they had baseline hypertension and diabetes, developed pregnancy-associated diabetes, or were diagnosed as having eclampsia or HELLP syndrome. The women with preeclampsia developed hypertension and proteinuria and had earlier deliveries as compared to the women with normal pregnancies. Using PLAP as the surrogate marker, pcEV were detected in peripheral blood sam- 
ples from all 17 pregnant women, but their levels were significantly higher in the preeclamptic patients than in the women with normal pregnancies at comparable gestational ages (Figure 1A). The levels of pcEV in patients with preeclampsia returned to the baseline during the postpartum period (Figure 1B).

This study of patients had a limited ability to investigate the underlying mechanisms of preeclampsia because it lacked manipulability of the clinical course and required extensive stratification of confounding clinical variables. To address these limitations, we conducted a mechanistic study in mouse models. We measured plasma levels of pcEV in pregnant mice using syncytin as the surrogate marker. Consistent with human data, plasma pcEV were detected in pregnant mice, reaching peak levels at 17-18 $\mathrm{dpc}$, and rapidly returning to baseline during the postpartum period (Figure 1C-F). PcEV detection by the syncytin antibody was further validated using another placental marker, endoglin (Online Supplementary Figure S1). The levels of syncytin on pcEV were closely correlated with the levels of annexin $\mathrm{V}^{+}$microvesicles $\left(\mathrm{R}^{2}=0.766, P<0.001\right)$ (Online Supplementary Figure S2), suggesting that most syncytin ${ }^{+}$pcEV expressed anionic phospholipids. However,

Table 1. Clinical information of the pregnant women included in the study.

\begin{tabular}{lccc} 
& PE & Normal pregnancy & P value* \\
Number & 10 & 7 & \\
Age (years) (mean \pm SD) & $33.1 \pm 4.2$ & $31.4 \pm 4.7$ & 0.219 \\
\hline Time of blood drawing (day) & $233 \pm 21$ & $233 \pm 20$ & 0.989 \\
Systolic BP (mmHg) & $166 \pm 14$ & $122 \pm 7$ & $<0.001$ \\
\hline Diastolic BP (mmHg) & $104 \pm 9$ & $77 \pm 8$ & $<0.001$ \\
Proteinuria (g/24h) & $5.37 \pm 4.4$ & $0.01+0.0$ & $<0.001$ \\
\hline Time of delivery (day)** & $239 \pm 23$ & $270 \pm 9$ & 0.003
\end{tabular}

*Student $t$-test; **Nine of the ten women with preeclampsia had a Cesarean delivery whereas all women with normal pregnancies had natural vaginal delivery. PE: preeclampsia; SD: standard deviation; BP: blood pressure.

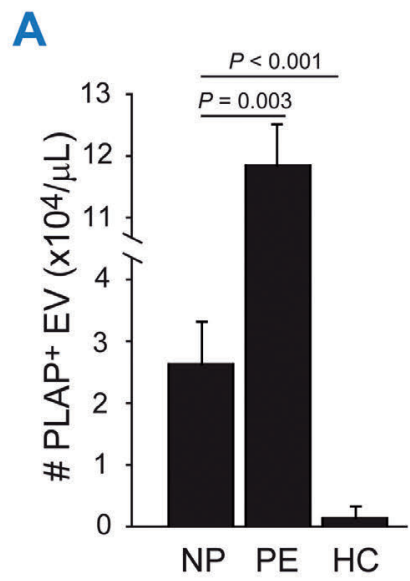

B

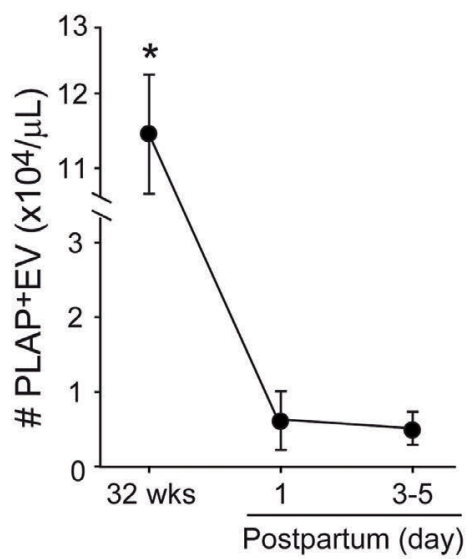

G

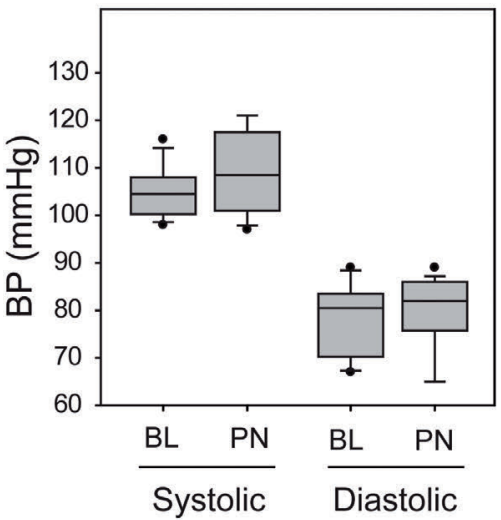

$\mathrm{H}$

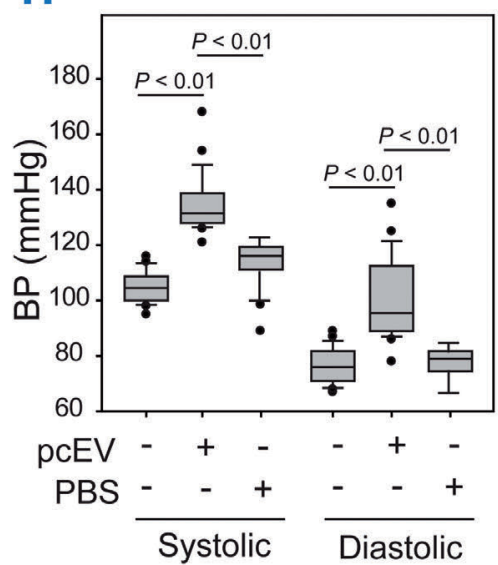

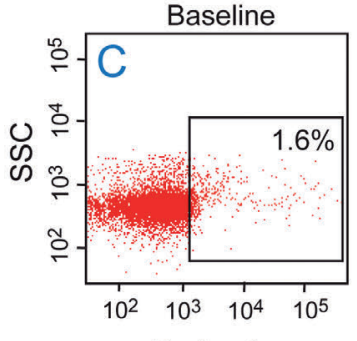
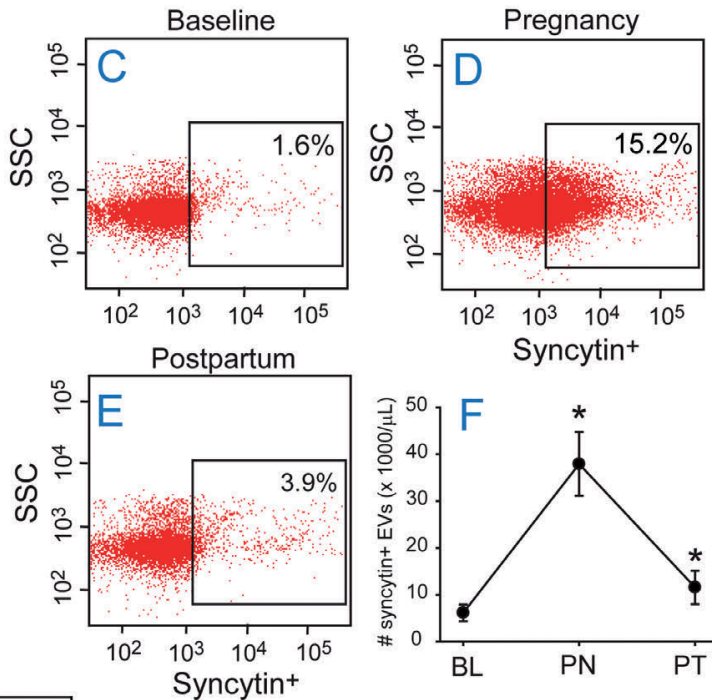

I

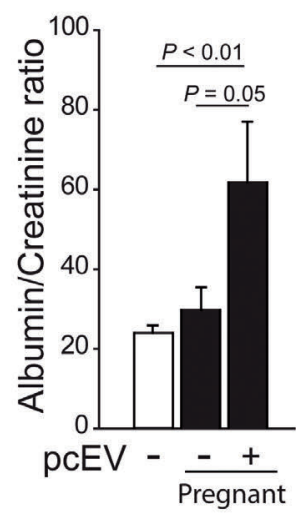

Figure 1. Placenta-derived extracellular vesicles in women with preeclampsia and in pregnant mice. (A) Plasma levels of placental alkaline phosphatase (PLAP) pcEV in women with normal pregnancy (NP, $n=7)$, patients with preeclampsia (PE, $n=10)$, and non-pregnant women $(H C, n=5)($ one-way analysis of variance, ANOVA). (B) Plasma levels of PLAP+ pcEV of PE patients at 32 weeks of pregnancy and during the postpartum period (one-way ANOVA with Tukey test, $n=10$, * $P<0.01$ vs. postpartum). Longitudinal changes of plasma syncytin ${ }^{+}$pcEV of pregnant mice at baseline (BL), at 17-18 days post-coitus (dpc) (PN) and postpartum (PT). (C-E): Cytometry dot plots from a pregnant mouse. (F) Summary from 15 mice (one-way ANOVA, ${ }^{*} P<0.001$, vs. BL and PT). (G) Blood pressure (BP) of C57BL/6J mice at $\mathrm{BL}$ and $17-18 \mathrm{dpc}\left(\mathrm{n}=30\right.$, paired t test). $(\mathrm{H}) \mathrm{BP}$ and (I) proteinuria of pregnant C57 BL/6J mice $(17-18 \mathrm{dpc})$ after infusion with $1 \times 10^{7} / \mathrm{mouse}$ of pcEV ( $\mathrm{m}=32$, one-way ANOVA). (J) Renal vascular leakage detected by an Evans blue extravasation test in pregnant $\mathrm{C} 57 \mathrm{BL} / 6 \mathrm{~J}$ mice infused with $1 \times 10^{7} \mathrm{pcEV} / \mathrm{mouse}$ or PBS $(\mathrm{n}=8$, paired $t$ test). 
these pregnant mice did not develop hypertension or proteinuria. The levels of circulating pcEV in pregnant mice reached peak levels of $3.8 \pm 0.9 \times 10^{7} / \mathrm{mL}$ (Figure $1 \mathrm{~F}$ ), which were comparable to those found in women with normal pregnancies $\left(2.6 \pm 1.3 \times 10^{7} / \mathrm{mL}\right)$, but significantly lower than those in the women with preeclampsia $\left(1.2 \pm 0.3 \times 10^{8} / \mathrm{mL}\right)$ (Figure 1A). We therefore intravenously infused pregnant mice with a single dose of pcEV $\left(1 \times 10^{7} /\right.$ mouse $)$ purified from injured placenta. The pcEV generated using this protocol were similar to those detected in the peripheral blood of pregnant mice in terms of size and syncytin expression, ${ }^{31}$ but they expressed a higher level of anionic phospholipids detected by annexin V (Online Supplementary Figure S3). The pregnant mice infused with injury-produced $\mathrm{pcEV}$ developed hypertension (Figure $1 \mathrm{H}$ ) 30 min after the infusion and proteinuria was detected in urine samples collected over $24 \mathrm{~h}$ after the pcEV infusion (Figure 1I). The hypertension was not caused by an expansion of volume due to the pcEV infusion (100 $\mu \mathrm{L} /$ mouse), as an equal volume of phosphate-buffered saline (PBS) induced minimal BP changes. Consistent with the development of proteinuria, vascular leakage was detected by Evans blue extravasation in the kidneys of pregnant mice infused with pcEV (Figure 1J). These data demonstrate that a high level of circulating
A
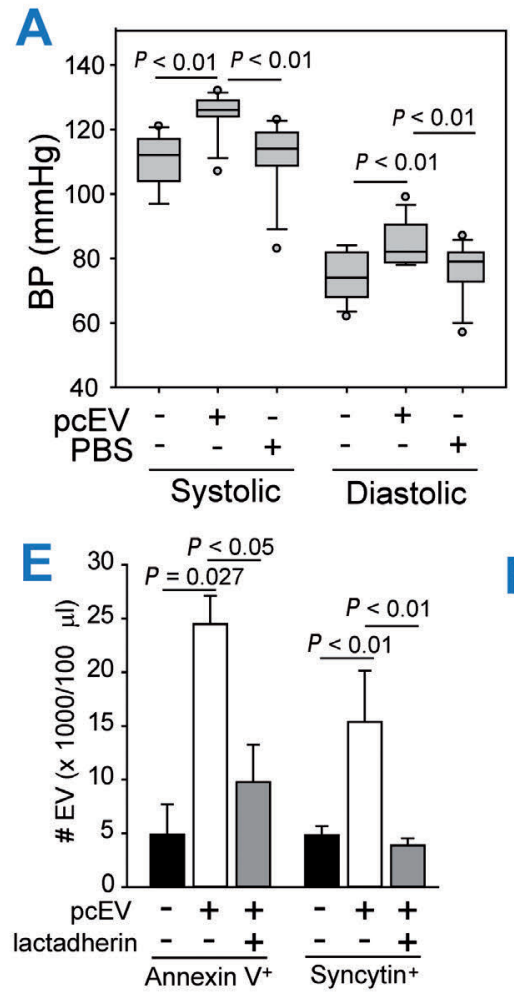

B

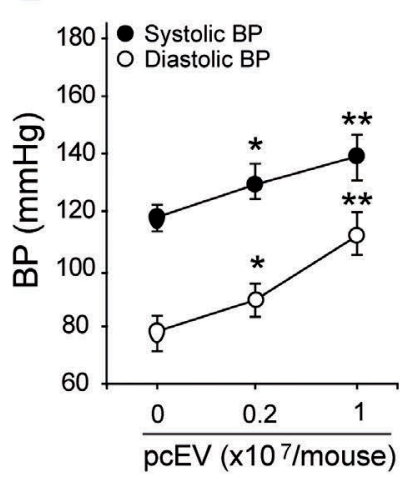

$\mathrm{F}$

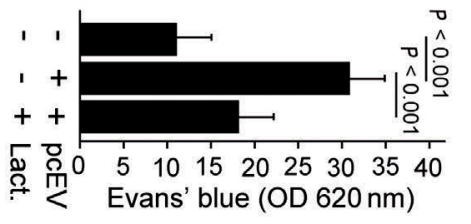

C

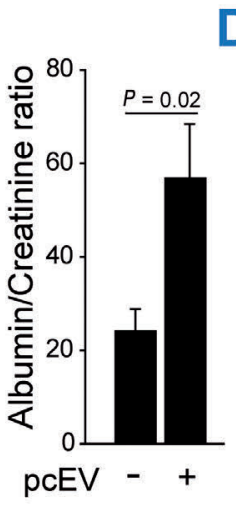

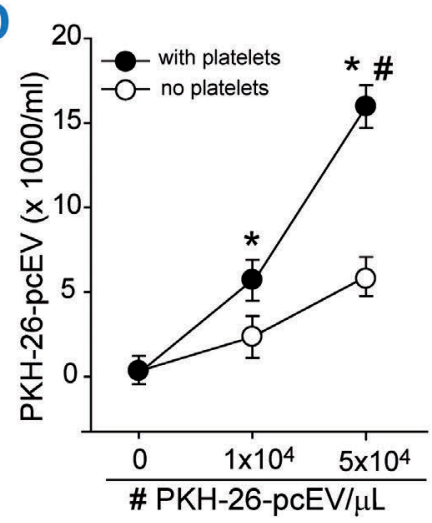

$G$

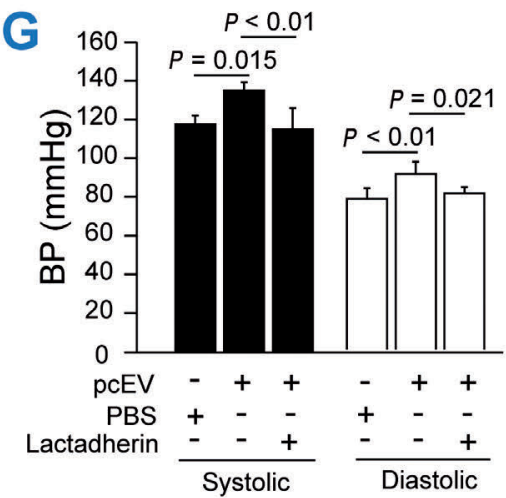

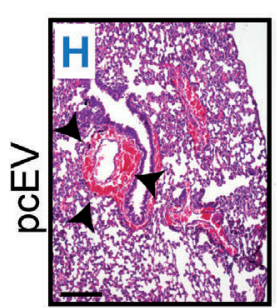

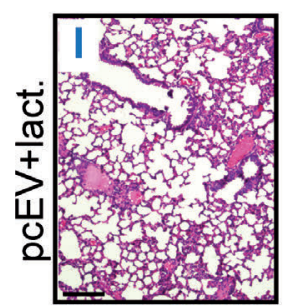

M

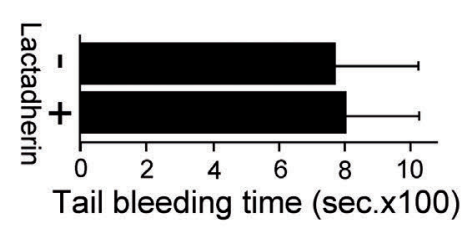

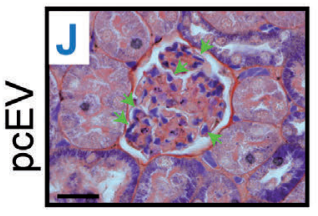
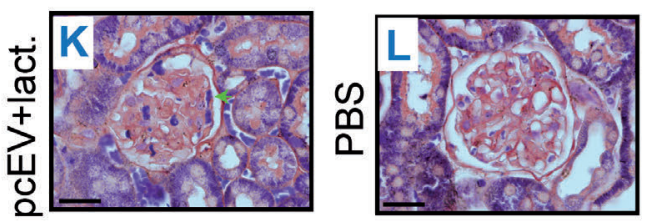

Figure 2. Placenta-derived extracellular vesicles induced a preeclampsia-like condition. Non-pregnant mice infused with $1 \times 10^{7} /$ mouse of injury-released pcEV developed (A) hypertension, $(B)$ in a dose-dependent manner, and (C) proteinuria $(n=18, A$ and $B$ : one-way ANOVA, $* P<0.05$ and $* * P<0.001$ vs. baseline; C: Student $t$-test). (D) Transendothelial migration of PKH-26-labeled pcEV in the presence and absence of platelets after $3 \mathrm{~h}$ of incubation $\left(n=26\right.$, one-way ANOVA, ${ }^{*} P<0.001$ vs. baseline, $\# P<0.001$ vs. no platelets). (E) Plasma levels of syncytin ${ }^{+}$and annexin $\mathrm{V}^{+}$extracellular vesicles, (F) Evans blue extravasation, and (G) blood pressure of mice infused with $1 \times 10^{7} \mathrm{pcEV} / \mathrm{mouse}$ and $400 \mu \mathrm{g} / \mathrm{kg}$ of lactadherin or pcEV alone ( $n=26$, one-way ANOVA). The lung tissue was sectioned and stained (Online Supplementary Methods). Perivascular bleeding and tissue edema of the lungs detected by staining with hematoxylin \& eosin (H-I, black arrows) and fibrin deposition in glomerular capillaries shown by phosphotungstic acid hematoxylin stain (J-L, green arrows) in pcEV-infused mice given lactadherin or phosphate-buffered saline (PBS) (representative images from 26 mice). (M) Tail bleeding of pcEV-infused mice given lactadherin or PBS ( $n=26$, Student $t$-test). BP: blood pressure; EV: extracellular vesicles; Lact: lactadherin; OD: optical density. 
pcEV from injured placenta could induce a preeclampsialike phenotype in pregnant mice.

\section{Placenta-derived extracellular vesicles directly induced hypertension and proteinuria}

To examine the effect of pcEV specifically, without the confounding influences of pregnancy, we infused pcEV from injured placenta into non-pregnant C57 BL/6J female mice. These mice developed acute hypertension $30 \mathrm{~min}$ after the pcEV infusion in a dose-dependent manner (Figure 2A, B) and proteinuria was detected in 24-h urine samples (Figure 2C). Furthermore, these pcEV disrupted the integrity of cultured endothelial cells, especially in the presence of platelets (Figure 2D). These data suggest that pcEV can directly induce a preeclampsia-like phenotype, independent of other pregnancy-induced changes.

We then investigated whether the microvesicle-scavenging factor lactadherin ${ }^{28}$ could prevent or reduce this
pcEV-induced preeclampsia-like condition. Mice infused with pcEV along with $400 \mu \mathrm{g} / \mathrm{kg}$ of lactadherin had lower levels of circulating annexin $\mathrm{V}^{+}$microvesicles, including pcEV (Figure 2E), reduced renal vascular leakage (Figure $2 \mathrm{~F}$ ), and did not develop hypertension (Figure 2G), in contrast to mice that received $\mathrm{pcEV}$ alone. Lactadherin also prevented the perivascular bleeding and tissue edema found in the lungs of pcEV-infused mice (Figure $2 \mathrm{H}$, I) and reduced the pcEV-induced hypercoagulable state that was defined by a shortened clotting time (Online Supplementary Figure S4A), platelet activation (Online Supplementary Figure $S 4 B$ ), the elevated level of annexin $\mathrm{V}^{+} \mathrm{EV}$ (Online Supplementary Figure S4C), and extensive fibrin deposition in the glomerular capillary (Figure 2J, K), without prolonging the tail bleeding time (Figure $2 \mathrm{M}$ ) or changing bloodcell counts and hematocrit (Online Supplementary Figure S5). The livers from mice infused with pcEV, but not those from mice infused with PBS, showed focal tissue necrosis
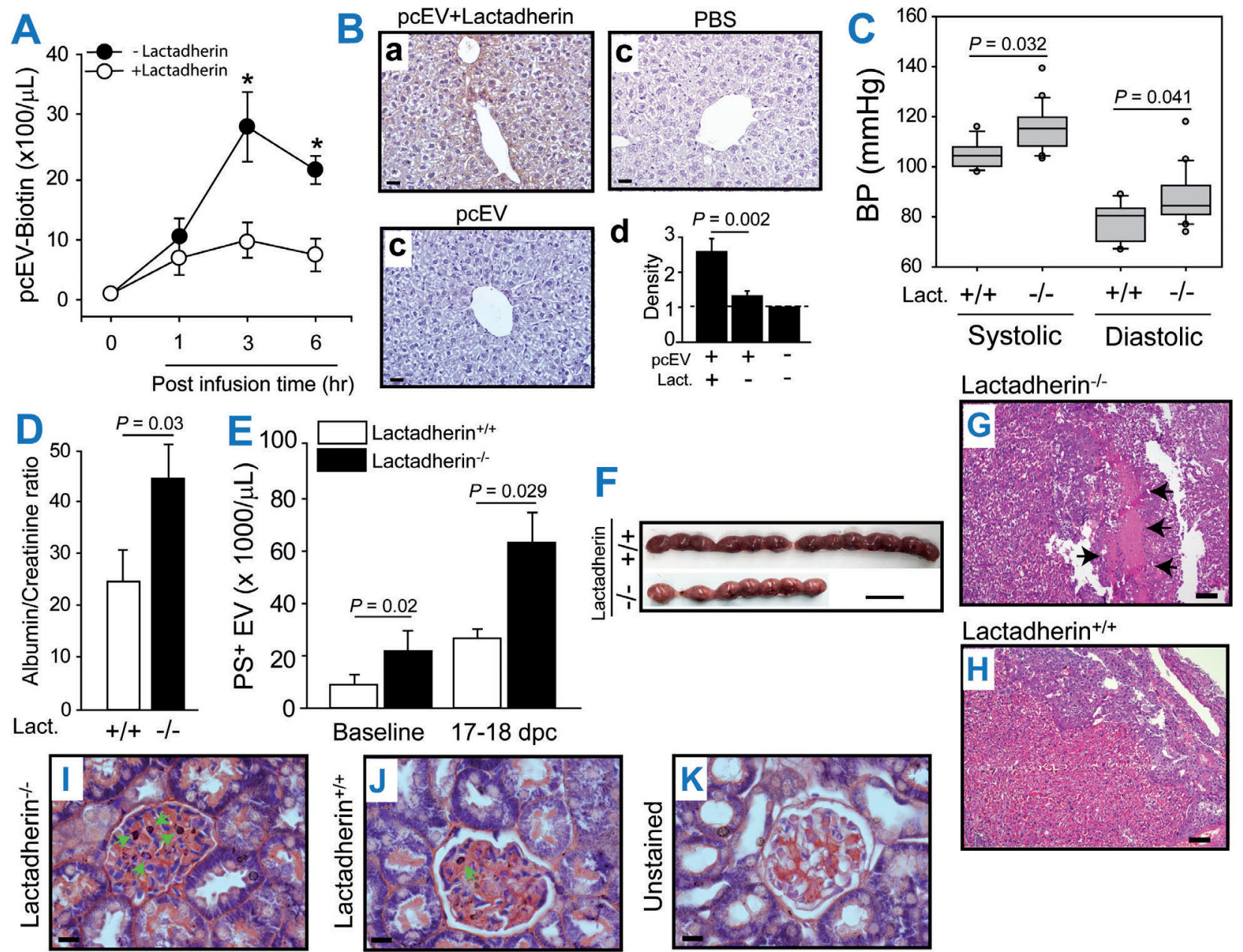

Figure 3. Prevention of a placenta-derived extracellular vesicle-induced preeclampsia-like condition by lactadherin. (A) Plasma levels of biotinylated pcEV in $\mathrm{C} 57 \mathrm{BL} / 6 \mathrm{~J}$ mice infused with $1 \times 107 /$ mouse of pcEV alone or combined with $400 \mu \mathrm{g} / \mathrm{kg}$ of lactadherin $[\mathrm{n}=10$, one-way analysis of variance (ANOVA), * $P<0.01 \mathrm{vs}$. baseline (BL)]. (B) Horseradish peroxidase streptavidin-stained liver tissue from mice infused with biotinylated pcEV alone, biotinylated pcEV in combination with lactadherin, and phosphate-buffered saline (a-c: representative images, d: optical densities of tissue scans, $n=19$, one-way ANOVA). (C) Blood pressure and (D) proteinuria of lactadherin ${ }^{--}$and lactadherin ${ }^{+/+}$mice measured at $17-18$ days post-coitus (dpc) $\left(n=8-24\right.$, Student $t$-test). (E) Plasma levels of phosphatidylserine $e^{+}$microvesicles measured at BL and 17-18 dpc ( $n=16$, Student $t$-test). (F) Placenta from lactadherin $\%$ and lactadherin ${ }^{+/+}$mice after $17-18 \mathrm{dpc}$. (G, H) Placenta at $17-18 \mathrm{dpc}$ from lactadherin $^{\prime-}(\mathrm{G})$, not lactadherin ${ }^{+/+}(\mathrm{H})$ mice showing tissue necrosis (black arrows, bar $\left.=100 \mu \mathrm{m}\right)$. (I, J) Phosphotungstic acid hematoxylin stain for fibrin deposition in the glomerular capillaries of lactadherin $\%$ mice (I, green arrows indicate fibrin) and not lactadherin ${ }^{+/+}$mice $(\mathrm{J})$. (K) Unstained background control. Images are representative of 39 mice. BP: blood pressure; PS: phosphatidylserine. 
and infiltration by inflammatory cells; these changes were not detected in pcEV-infused mice that also received lactadherin (Online Supplementary Figure S6). In contrast to kidney tissues, intravascular fibrin deposition was very limited in the livers of pcEV-infused mice.

To measure directly whether lactadherin enhanced pcEV clearance, biotinylated pcEV $\left(1 \times 10^{7} /\right.$ mouse $)$ were infused into non-pregnant C57BL/6J mice along with 400 $\mu \mathrm{g} / \mathrm{kg}$ of lactadherin or an equal volume of PBS. The plasma level of biotinylated pcEV reached a plateau $3 \mathrm{~h}$ after infusion (Figure 3A) but was significantly lower in mice that also received lactadherin (Figure $3 \mathrm{~A}$ ). The mice given pcEV and lactadherin had more extensive accumulation of biotinylated pcEV in their livers (Figure $3 \mathrm{~B}$ ).

\section{Lactadherin-deficient mice developed unprovoked preeclampsia during pregnancy}

To further validate the role of lactadherin in promoting EV clearance and preventing preeclampsia, we also exam- ined lactadherin ${ }^{-1}$ mice and their wildtype littermates (Online Supplementary Figure S7). At 17-18 dpc, lactadherin mice had higher BP (Figure 3C) and developed proteinuria (Figure 3D) without pcEV infusion, as required for pregnant C57BL/6 mice (Figure 1H). Plasma levels of PS-expressing EV recognized by annexin $\mathrm{V}$ were significantly higher in lactadherin $^{-/}$mice than in their wildtype littermates at baseline, and they increased further during pregnancy (Figure $3 \mathrm{E})$. There was no visible difference in the appearance of the fetuses and sizes of placenta between lactadherin ${ }^{-/}$and lactadherin ${ }^{+/+}$mice at 17-18 dpc (Online Supplement Figure S8), but the number of lactadherin ${ }^{-1}$ litters was significantly less than the number of lactadherin ${ }^{+/+}$litters $(8.2 \pm 1.7 \mathrm{vs}$. $11.5 \pm 1.4$, Student $t$-test, $P<0.05$ ) (Figure 3F). Hematoxylin \& eosin stains of placental tissues frequently detected tissue necrosis in the trophoblast villi and decidua of lactadherin mice (Figure 3G,H). The lactadherin deficiency also resulted in more extensive fibrin deposition in glomerular capillaries, which occurred less in wildtype littermates (Figure 3I-K).
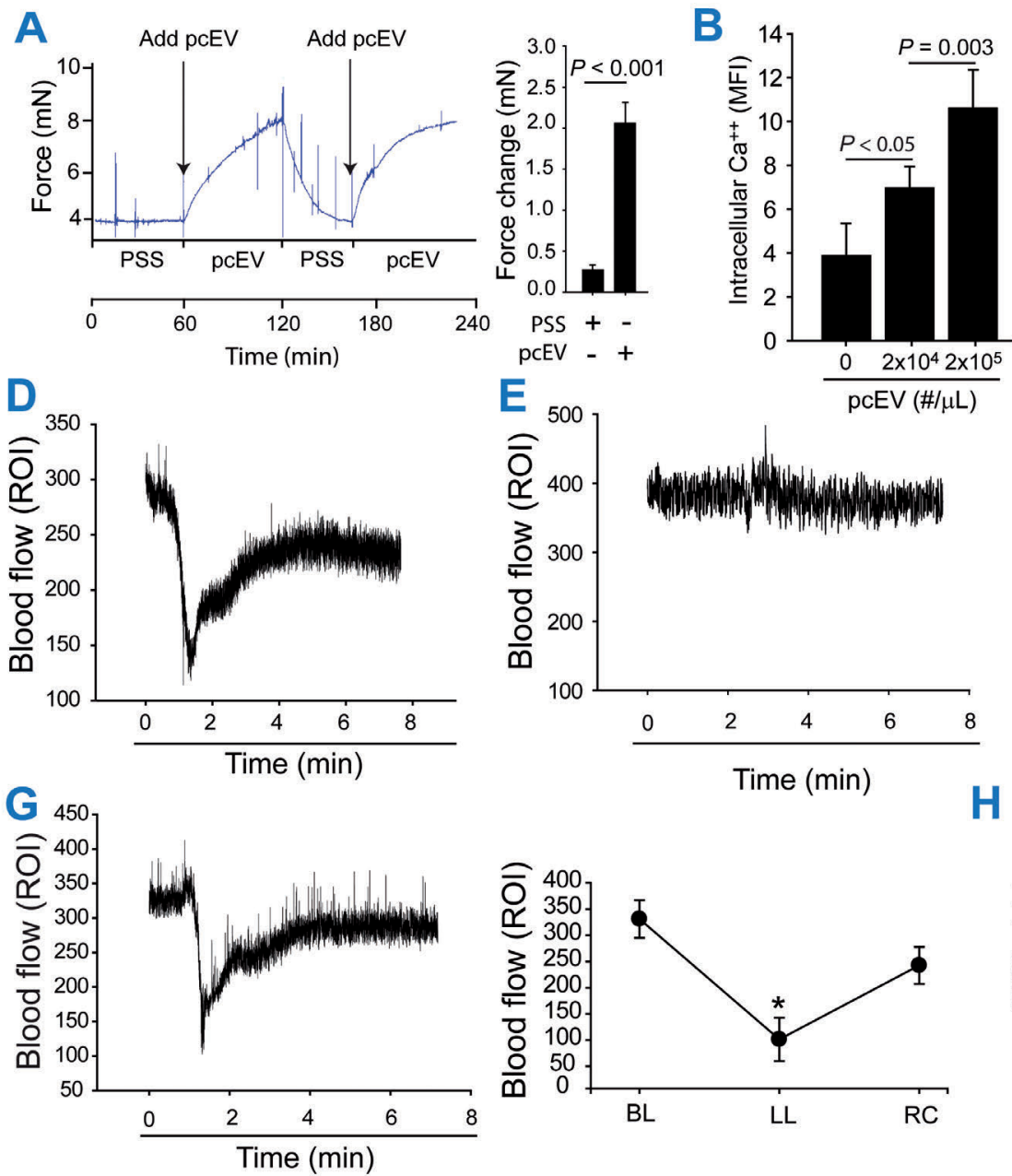
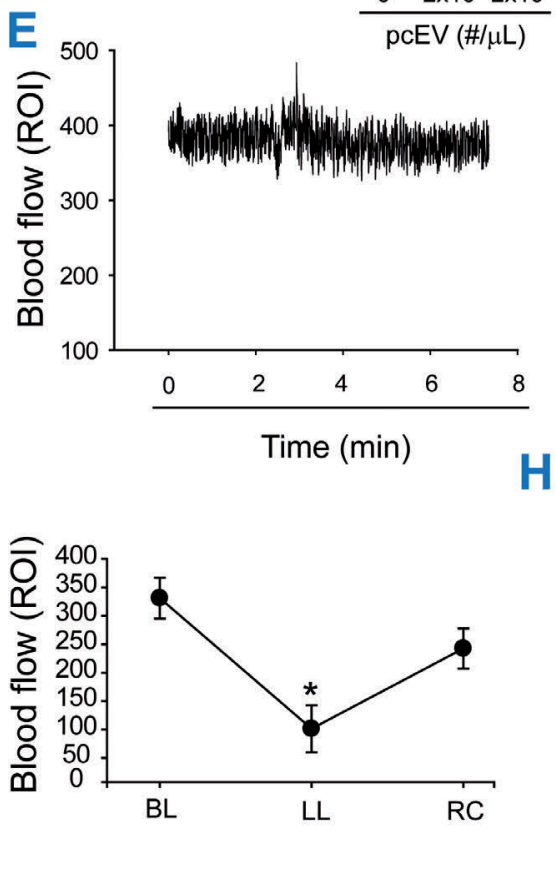

$\mathrm{H}$
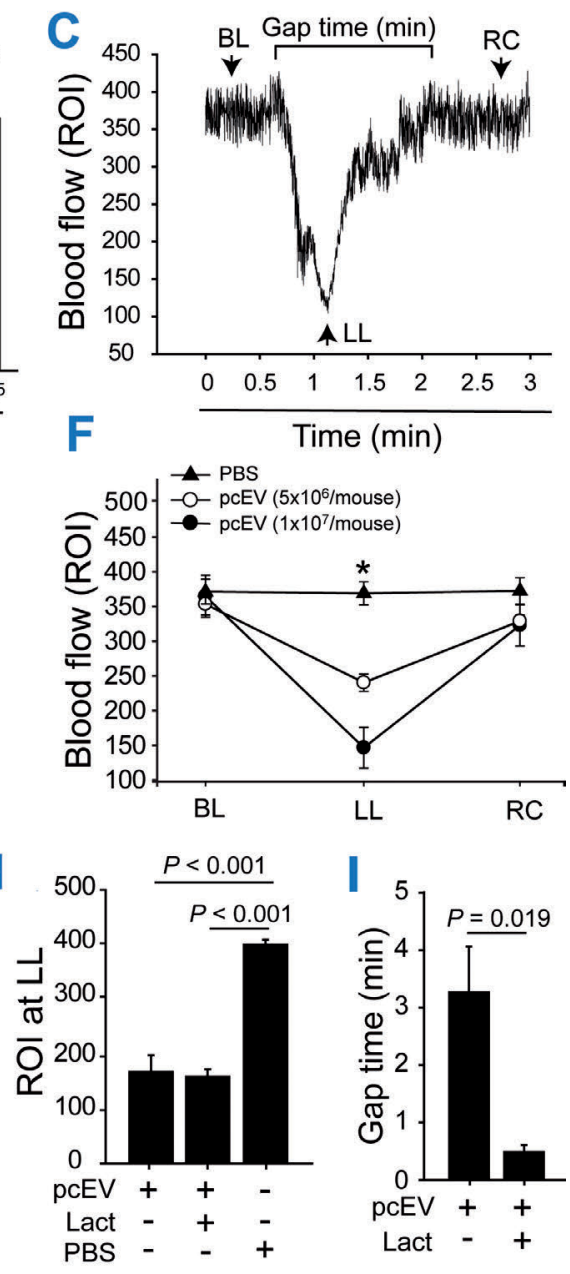

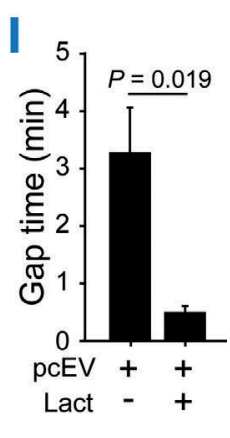

Figure 4. Placenta-derived extracellular vesicles induced vasoconstriction. (A) Placenta-derived extracellular vesicles $(\mathrm{pcEV})\left(2 \times 10^{5} / \mu \mathrm{L}\right)$ increase the vascular ten sion of the carotid artery (left: a myograph of pcEV-induced vasoconstriction; right: summary of effects in 20 experiments, Student t-test). (B) Calcium influx of cultured smooth muscle cells treated with pcEV $\left[n=12\right.$, one-way analysis of variance (ANOVA)]. (C-F) pcEV $\left(1 \times 10^{7} /\right.$ mouse) but not phosphate-buffered saline (PBS) rapidly reduced cerebral blood flow as determined by laser speckle contrast analysis (LASCA) (BL: baseline; LL: lowest level, gap-time of flow reduction; and RC: flow recovery). The reduction is either fully (C) or partially (D) recovered (C-E: representatives graphs; F: summary of 12 experiments, one-way ANOVA). (G) The cerebral blood flow of mice receiving extracellular vesicles purified from patients with preeclampsia (top: a representative graph, bottom: summary of 3 experiments, oneway ANOVA, ${ }^{*} P<0.001 \mathrm{vs}$. BL). The LL values $(\mathrm{H})$ and gap-time (I) of pcEV-infused mice that also received $400 \mu \mathrm{g} / \mathrm{kg}$ lactadherin or PBS ( $\mathrm{n}=12$, one-way ANOVA on ranks). ROI: region of interest; Lact: lactadherin. 
Taken together, these results demonstrate that enhancing EV clearance prevented pcEV-induced hypertension, vascular leakage, and hypercoagulation.

\section{Placenta-derived extracellular vesicles induced vasoconstriction}

We used three complementary experiments to investigate how pcEV induce hypertension. First, pcEV increased the wall tension of the carotid artery (Figure 4A). The vasoconstriction was relaxed to the baseline level upon removal of pcEV and was induced again after reincubation with pcEV. Second, pcEV triggered the calcium influx of cultured smooth-muscle cells in a dose-dependent manner (Figure 4B). Third, a single-dose infusion of pcEV but not PBS induced a rapid and dose-dependent reduction of cerebral blood flow (Figure 4C-F). The reduction was transient, and the blood flow quickly recovered to either the pre-infusion level (Figure 4C) or a persistently low level (Figure 4D). This pcEV-induced reduction in blood flow was also detected in mice infused with total EV purified from plasma of patients with preeclampsia (Figure 4G). Lactadherin $(400 \mu \mathrm{g} / \mathrm{kg})$ given together with pcEV did not prevent the reduction of blood flow (Figure $4 \mathrm{H}$ ) but significantly shortened its duration (Figure 4I). These data demonstrate that $\mathrm{pcEV}$ directly induced vasoconstriction by mobilizing intracellular calcium, resulting in the systemic reduction in microvascular blood flow.

\section{Discussion}

We have investigated the role of pcEV in the pathogenesis of preeclampsia by examining plasma samples from preeclamptic women, studying mouse models, and conducting in vitro experiments. Consistent with previous reports, ${ }^{10,11}$ we detected pcEV in blood samples of women in the late stage of pregnancy, but pcEV levels were significantly higher in patients with preeclampsia (Figure $1 \mathrm{~A})$ and rapidly returned to baseline during the postpartum period (Figure 1B). These clinical findings led us to study the activity of pcEV in new mouse models. These new mouse models offered advantages over previously used models because they did not require surgery, genetic manipulation, or pharmacological interventions. We made several novel observations that collectively define a causal role of pcEV in the pathogenesis of preeclampsia and a means to prevent it.

First, pregnant mice developed hypertension and proteinuria only after intravenous infusion of pcEV from injured placenta (Figures $1 \mathrm{H}$ ), suggesting that preeclampsia can be induced either by a high level of circulating pcEV or by pcEV derived from injured placenta. Our results support the latter possibility because pcEV from injured placenta caused a preeclampsia-like condition in non-pregnant mice at a significantly lower number of $1 \times 10^{7}$ vesicles/mouse (Figure $2 \mathrm{~A}, \mathrm{~B}$ ) than the $3.8 \pm 0.9 \times 10^{7}$ vesicles/mouse found in pregnant mice (Figure 1F). These pcEV from injured placenta were similar in size and syncytin expression to those found in pregnant mice, but expressed a significantly highly level of anionic phospholipids recognized by annexin $\mathrm{V}$. These anionic phospholipids were strongly procoagulant and could also disrupt the integrity of the endothelium. In addition, syncytiotrophoblast EV from women with preeclampsia carry less endothelial nitric oxide synthase compared to those from women with normal pregnancies ${ }^{25}$ and thus have a reduced synthesis of the vasodilating factor nitric oxide. The fast development of hypertension in non-pregnant mice infused with pcEV (30 min after infusion) (Figures 2 and 3 ) is likely caused by the quick infusion of a large quantity of pcEV ( $<5 \mathrm{~min})$. In contrast, pcEV are probably released gradually during pregnancy and induce hypertension when they reach a critical threshold level in the circulation over a longer period of time.

Second, pcEV disrupted the endothelial barrier in vitro, especially in the presence of platelets to induce vascular leakage (Figure 2D), which could be responsible for pcEVinduced proteinuria (Figures 1I and 2C). The synergistic activity between pcEV and platelets is consistent with a recent study showing that $\mathrm{EV}$ activate maternal platelets to promote inflammation and a preeclampsia-like patholo$\mathrm{gy}^{32}$ but how platelets enhance pcEV-induced endothelial injury remains to be investigated. Nevertheless, the pcEVinduced endothelial injury not only results in vascular leakage, but could also contribute to the development of hypertension by impairing the endothelium-dependent vasodilation machinery (e.g., endothelial nitric oxide synthase nitric oxide pathway) ${ }^{30,33}$ and allowing pcEV to act directly on smooth muscle to trigger calcium-dependent vasoconstriction (Figure 4). Several calcium signaling pathways are involved in regulating smooth-muscle contractility, ${ }^{34,35}$ but the molecule(s) on pcEV that triggers one or all of these pathways remains to be identified. This vasoconstrictive activity is unlikely to be limited to pcEV because EV from injured endothelial cells also induce hypertension in pregnant mice in a platelet-dependent manner. ${ }^{32}$ The pcEVinduced vasoconstriction reduced blood flow (Figure 4C-F), potentially resulting in tissue ischemia that further propagates injury to the placenta and the endothelium.

Third, pcEV induced a systemic hypercoagulable state defined by shortened clotting time, platelet activation, and the expression of procoagulant PS (Online Supplementary Figure S4). This hypercoagulable state has been widely reported in patients with preeclampsia, and causes extensive fibrin deposition in glomerular vessels (Figure 2J-L). The fibrin deposition could potentially induce vascular leakage and increase rigidity of the vessel wall, contributing to pcEV-induced renal dysfunction (i.e., proteinuria) and hypertension, respectively. ${ }^{38}$ Surprisingly, fibrin deposition may be organ-specific because it was extensively detected in the kidney, but minimally in the liver vasculature (Online Supplementary Figure S6). Instead, focal tissue necrosis and infiltration of inflammatory cells were detected in the liver of pcEV-infused mice, but the cause of this tissue injury remains to be identified. Nevertheless, our findings are consistent with those of a recent cohort study, which showed that plasma samples from preeclamptic patients had elevated levels of EV from activated platelets and leukocytes as well as tissue factorbearing EV, as compared to women with normal pregnancies. ${ }^{39}$ However, another study found no association between preeclampsia and plasma levels of anionic phospholipid-expressing EV. ${ }^{40}$

Finally, we have recently shown that lactadherin given intravenously promotes the phagocytosis of EV by coupling the vesicles to macrophages in the liver through their PS- and integrin-binding domains. ${ }^{28}$ Here, we further show that lactadherin given intravenously also promotes the phagocytosis of pcEV (Figure 3A, B) and prevents pcEV-induced hypertension, proteinuria, and coagulation 
(Figure 2). Lactadherin ${ }^{-/}$mice have elevated levels of PSexpressing EV (Figure 3E) and develop hypertension and proteinuria during pregnancy (Figure 3C, D). EV found in lactadherin ${ }^{-1}$ mice before pregnancy likely come from platelets and endothelial cells, and could further propagate endothelial and placental injury, systemic inflammation, and coagulation. ${ }^{32,41}$ The findings in mice infused with exogenous lactadherin and those deficient in lactadherin raise several questions, (i) Could insufficient EV clearance induce placenta damage? (ii) Is intrinsically low EV-clearance activity a risk for preeclampsia and, if so, could it serve as a predictive marker? (iii) Could lactadherin be used as a treatment for preeclampsia? Human plasma contains $\sim 1 \mathrm{ng} / \mathrm{mL}$ of lactadherin,$^{42}$ which may be sufficient during homeostasis, but becomes insufficient to remove a large quantity of microvesicles that are substan- tially and persistently released during pregnancy, especially in the condition of preeclampsia.

In summary, we demonstrated that pcEV from injured placenta induced a preeclampsia-like condition in mice by inducing endothelial injury, vasoconstriction, and hypercoagulation. This $\mathrm{pcEV}$-induced condition was prevented by enhancing EV clearance. The rates of $\mathrm{pcEV}$ production and clearance could therefore be used for the risk assessment of preeclampsia and become new therapeutic targets for preeclampsia.

\section{Funding}

This study was supported by National Institutes of Health grants NS087296, HL119391 and HL125957 (to JFD), Natural Science Foundation of China State Key Program grant 81330029 (to JNZ) and research grant 81672399 (to ML).

\section{References}

1. Tranquilli AL, Dekker G, Magee L, et al. The classification, diagnosis and management of the hypertensive disorders of pregnancy: a revised statement from the ISSHP. Pregnancy Hypertens. 2014;4(2):97-104.

2. Phoa KY, Chedraui P, Perez-Lopez FR, et al. Perinatal outcome in singleton pregnancies complicated with preeclampsia and eclampsia in Ecuador. J Obstet Gynaecol. 2016;36(5):581-584

3. Redman CW, Tannetta DS, Dragovic RA, et al. Review: does size matter? Placental debris and the pathophysiology of preeclampsia. Placenta. 2012;33(Suppl):S48-54.

4. Amaral LM, Wallace K, Owens M, LaMarca B. Pathophysiology and current clinical management of preeclampsia. Curr Hypertens Rep. 2017;19(8):61

5. Chaiworapongsa T, Chaemsaithong P, Yeo L, Romero R. Pre-eclampsia part 1: current understanding of its pathophysiology. Nat Rev Nephrol. 2014;10(8):466-480

6. Lok CA, Van der Post JA, Sturk A, Sargent IL, Nieuwland R. The functions of microparticles in preeclampsia. Pregnancy Hypertens. 2011;1(1):59-65.

7. Guller S, Tang Z, Ma YY, Di Santo S, Sager $\mathrm{R}$, Schneider $\mathrm{H}$. Protein composition of microparticles shed from human placenta during placental perfusion: potential role in angiogenesis and fibrinolysis in preeclampsia. Placenta. 2011;32(1):63-69.

8. Rusterholz C, Messerli M, Hoesli I, Hahn S. Placental microparticles, DNA, and RNA in preeclampsia. Hypertens Pregnancy. 2011;30 (3):364-375

9. Mincheva-Nilsson L, Baranov V. Placentaderived exosomes and syncytiotrophoblast microparticles and their role in human reproduction: immune modulation for pregnancy success. Am J Reprod Immunol. 2014;72(5):440-457.

10. Germain SJ, Sacks GP, Sooranna SR, Sargent IL, Redman CW. Systemic inflammatory priming in normal pregnancy and preeclampsia: the role of circulating syncytiotrophoblast microparticles. J Immunol. 2007:178(9):5949-5956.

11. Goswami D, Tannetta DS, Magee LA, et al. Excess syncytiotrophoblast microparticle shedding is a feature of early-onset preeclampsia, but not normotensive intrauterine growth restriction. Placenta. 2006;27(1): 56-61.

12. Dragovic RA, Collett GP, Hole P, et al. Isolation of syncytiotrophoblast microvesicles and exosomes and their characterisation by multicolour flow cytometry and fluorescence nanoparticle tracking analysis. Methods. 2015;87:64-74.

13. Baig S, Kothandaraman N, Manikandan J, et al. Proteomic analysis of human placental syncytiotrophoblast microvesicles in preeclampsia. Clin Proteomics. 2014;11 (1): 40 .

14. Nakashima A, Yamanaka-Tatematsu M, Fujita N, et al. Impaired autophagy by soluble endoglin, under physiological hypoxia in early pregnant period, is involved in poor placentation in preeclampsia. Autophagy. 2013:9(3):303-316.

15. Velicky P, Windsperger K, Petroczi K, et al. Pregnancy-associated diamine oxidase originates from extravillous trophoblasts and is decreased in early-onset preeclampsia. Sci Rep. 2018;8(1):6342.

16. Saito S, Nakashima A. A review of the mechanism for poor placentation in earlyonset preeclampsia: the role of autophagy in trophoblast invasion and vascular remodeling. J Reprod Immunol. 2014;101-102:80-88.

17. Rusterholz C, Holzgreve W, Hahn S. Oxidative stress alters the integrity of cellfree mRNA fragments associated with placenta-derived syncytiotrophoblast microparticles. Fetal Diagn Ther. 2007;22(4): 313-317.

18. Brown CE, Flynn J, Carty DM, Scotland G Delles C. Lb01.05: Vascular consequences of pre-eclampsia. J Hypertens. 2015;33(Suppl 1):e46.

19. Cooper JC. The effect of placental syncytiotrophoblast microvillous membranes from normal and pre-eclamptic women on the growth of endothelial cells in vitro. Br J Obstet Gynaecol. 1994;101(6):559.

20. Smarason AK, Sargent IL, Starkey PM Redman CW. The effect of placental syncytiotrophoblast microvillous membranes from normal and pre-eclamptic women on the growth of endothelial cells in vitro. $\mathrm{Br}$ ] Obstet Gynaecol. 1993;100(10):943-949.

21. Al-Ofi E, Anumba DO, Coffelt S. OS006.
Functional expression of endogenous ligands of Toll like receptor4 on monocytes and placentae from women during normal pregnancy andpre-eclampsia. Pregnancy Hypertens. 2012;2(3):178.

22. Bobek G, Surmon L, Mirabito KM, Makris A, Hennessy A. Placental regulation of inflammation and hypoxia after TNF-alpha infusion in mice. Am J Reprod Immunol. 2015;74(5):407-418.

23. Sergeeva ON, Chesnokova NP, Ponukalina EV, Rogozhina IE, Glukhova TN [Pathogenetic relationship between endothelial dysfunction and disorders of blood coagulation potential in pregnancy complicated by pre-eclampsia]. Vestn Ross Akad Med Nauk. 2015(5):599-603

24. Doridot L, Passet B, Mehats C, et al Preeclampsia-like symptoms induced in mice by fetoplacental expression of STOX1 are reversed by aspirin treatment. Hypertension. 2013;61(3):662-668.

25. Motta-Mejia C, Kandzija N, Zhang W, et al Placental vesicles carry active endothelial nitric oxide synthase and their activity is reduced in preeclampsia. Hypertension. 2017;70(2):372-381

26. Skynner MJ, Drage DJ, Dean WL, Turner S Watt DJ, Allen ND. Transgenic mice ubiquitously expressing human placental alkaline phosphatase (PLAP): an additional reporter gene for use in tandem with beta-galactosidase (lacZ). Int J Dev Biol. 1999:43(1):85-90.

27. Tian Y, Salsbery B, Wang M, et al. Brainderived microparticles induce systemic coagulation in a murine model of traumatic brain injury. Blood. 2015;125(13):2151-2159.

28. Zhou Y, Cai W, Zhao Z, et al. Lactadherin promotes microvesicle clearance to prevent coagulopathy and improves survival of severe TBI mice. Blood. 2018;131(5):563-572.

29. Zhao Z, Wang M, Tian Y, et al. Cardiolipinmediated procoagulant activity of mitochondria contributes to traumatic brain injury-associated coagulopathy in mice. Blood. 2016;127(22):2763-2772.

30. Pascoal IF, Lindheimer MD, NalbantianBrandt C, Umans JG. Preeclampsia selectively impairs endothelium-dependent relaxation and leads to oscillatory activity in small omental arteries. J Clin Invest. 1998;101(2):464-470.

31. Dupressoir A, Vernochet C, Bawa O, et al 
Syncytin-A knockout mice demonstrate the critical role in placentation of a fusogenic, endogenous retrovirus-derived, envelope gene. Proc Natl Acad Sci U S A. 2009;106(29):12127-12132.

32. Kohli S, Ranjan S, Hoffmann J, et al. Maternal extracellular vesicles and platelets promote preeclampsia via inflammasome activation in trophoblasts. Blood. 2016;128 (17):2153-2164

33. Pascoal IF, Umans JG. Effect of pregnancy on mechanisms of relaxation in human omental microvessels. Hypertension. 1996;28(2): 183-187.

34. Earley S, Brayden JE. Transient receptor potential channels in the vasculature. Physiol Rev. 2015;95(2):645-690.

35. Ghigo A, Laffargue M, Li M, Hirsch E. PI3K and calcium signaling in cardiovascular disease. Circ Res. 2017;121(3):282-292.

36. Bonnar J, McNicol GP, Douglas AS. Coagulation and fibrinolytic systems in preeclampsia and eclampsia. Br Med J. 1971;2(5752):12-16.

37. Erez O, Romero R, Vaisbuch E, et al. Tissue factor activity in women with preeclampsia or SGA: a potential explanation for the excessive thrombin generation in these syndromes. J Matern Fetal Neonatal Med. 2018;31(12):1568-1577.

38. Holdsworth SR, Tipping PG. Macrophageinduced glomerular fibrin deposition in experimental glomerulonephritis in the rabbit. J Clin Invest. 1985;76(4):1367-1374

39. Campello E, Spiezia L, Radu CM, et al. Circulating microparticles in umbilical cord blood in normal pregnancy and pregnancy with preeclampsia. Thromb Res. 2015;136 (2):427-431.

40. Katzenell S, Shomer E, Zipori Y, Zylberfisz A, Brenner B, Aharon A. Characterization of negatively charged phospholipids and cell origin of microparticles in women with gestational vascular complications. Thromb Res. 2012;130(3):479-484.

41. Owens AP 3rd, Mackman N. Microparticles in hemostasis and thrombosis. Circ Res. 2011;108(10):1284-1297.

42. Kishi C, Motegi SI, Ishikawa O. Elevated serum MFG-E8 level is possibly associated with the presence of high-intensity cerebral lesions on magnetic resonance imaging in patients with systemic lupus erythematosus. J Dermatol. 2017;44(7):783-788. 